\title{
Slotted Waveguide Array Antenna Performance Enhancement by Parasitic Elements Augmentation
}

\author{
Saad I. Alhuwaimel ${ }^{1}$ \\ Department of Electronic and Electrical Engineering, \\ University College London - Torrington Place \\ London, WC1E 7JE \\ United Kingdom \\ e-mail: huwaimel@kacst.edu.sa \\ saad.alhuwaimel@ucl.ac.uk
}

\author{
Kin-Fai Tong \\ Department of Electronic and Electrical Engineering, \\ University College London - Torrington Place \\ London, WC1E 7JE \\ United Kingdom \\ e-mail: k.tong@ucl.ac.uk
}

\begin{abstract}
A novel design of Slotted Waveguide Array Antenna (SWGAA), at $8.5 \mathrm{GHz}$ with beamwidth of $11^{\circ}$ and $>-21$ $\mathrm{dB}$ sidelobe level, with each slot is augmented with a pair of inverted L-shaped parasitic dipoles is presented in this work. Each dipole is located beside the long edges of the slot. The slot cut from a rectangular waveguide acts as a magnetic dipole while the parasitic dipoles act as electric dipoles. Both dipole types act as radiating elements. If both are designed to radiate at the same frequency at the proper amplitude and phase, the radiation performance can be enhanced. Performance comparisons between conventional SWGAA design and the proposed design are presented in this work.
\end{abstract}

Keywords-Waveguide, Slot, SWGAA, Parasitic element, Sidelobe Level, Front-to-Back Ratio.

\section{INTRODUCTION}

Utilizing slots cut in a rectangular waveguide walls as radiating elements started during World War II [1] [2]. The work reported at that time was done by Watson [2], Stevenson [3] and Booker [4]. Recently, several novel SWGAAs have been developed for different applications, e.g. Radar.

The SWGAAs are attractive antenna option as they have relatively simple design, light weight, small size as frequency increases, high power handling, high directivity and hence high gain. These features make the SWGAAs attractive especially in high power applications.

A single element standing-wave SWGAA azimuth beamwidth is mainly controlled by the number of slots in the waveguide wall. On the other hand, it has a fan elevation beamwidth. The sidelobe level of a conventional SWGAA, slots with equal offset from the rectangular waveguide broad-wall center line, is around $-13 \mathrm{~dB}$. The sidelobe level can largely be suppressed by tapering slots locations around the broad-wall center line. In addition, the mutual coupling between the slots in a SWGAA is considerably high with its highest level occurred between the two middle slots for an end-fed standing wave SWGAA.

Augmenting each slot by a pair of inverted L-shaped parasitic elements, dipoles, as shown in Fig. 4, was first theoretically discussed by Black and Clavin [5]. It was suggested

${ }^{1}$ King Abdulaziz City for Science and Technology, Kingdom of Saudi Arabia P.O Box 6086, Riyadh 11442 that this augmentation will enhance the SWGAA overall performance by improving its Front-to-Back $(\mathrm{F} / \mathrm{B})$ ratio and reducing the mutual coupling between slots.

The slots act as magnetic dipole while the parasitic elements act as electric dipoles. The idea of using the combination of both electric and magnetic dipoles was first described by [6] and Clavin, Huebner and Kilburg [7].

This work presents a novel SWGAA with parasitic dipoles that enhances the antenna gain, reduces the energy in the antenna backlobe, hugely decreases the mutual coupling between slots and narrows the antenna elevation beamwidth compared to a conventional SWGAA. The simulated results using the Computer Simulation Technology (CST) [8] are presented and discussed.

\section{X-BAND SWGAA}

\section{A. Conventional SWGAA}

Commercially available WR90/WG16 waveguide is used in designing the linearly polarized X-band SWGAA. This waveguide has an operating frequency range of $8.2-12.4 \mathrm{GHz}$. The waveguide internal dimensions are: $a=22.86 \mathrm{~mm}$ and $b=10.16 \mathrm{~mm}$ and are indicated in Fig. 1. The wall thickness used for the waveguide is $1.125 \mathrm{~mm}$ in the design as it is commercially available.

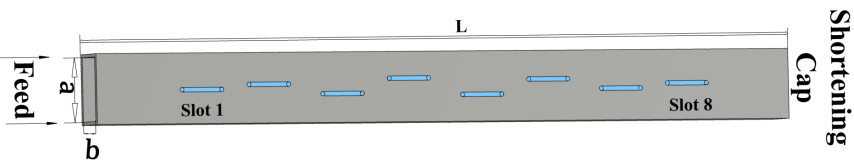

Fig. 1 Low sidelobe SWGAA

From [2] and [3], the spacing between the feeding point and the first slot and the last slot and the shortening cap is an odd multiple of quarter of the guide wavelength, as shown in Fig. 2. The wavelength of the wave propagating inside the wave guide, guide wavelength, is given by: 


$$
\lambda_{g}=\frac{1}{\sqrt{\left(\frac{1}{\lambda}\right)^{2}-\left(\frac{1}{2 a}\right)^{2}}}
$$

Elliot [9] modified the slot normalized conductance proposed by [3] that is used to calculate the slots offset from the waveguide broad-wall center line. The normalized conductance is given by:

$$
\begin{aligned}
\frac{G_{\text {slot }}}{G_{\text {waveguide }}}=2.09 \frac{\lambda_{g}}{\lambda} \frac{a}{b}\left[\cos \left(\frac{0.464 \pi}{\lambda_{g}}\right)\right. \\
-\cos (0.464 \pi)]^{2} \sin ^{2}\left(\frac{\pi x_{1}}{a}\right)
\end{aligned}
$$

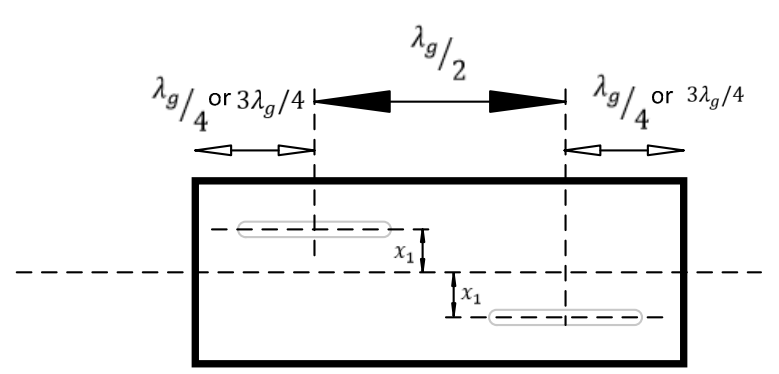

Fig. 2 Slots spacing

Single waveguide SWGAA with eight slots has achieved the desired azimuth beamwidth $\left(\approx 11.2^{\circ}\right)$. The vertical beamwidth is about $87^{\circ}$. The uniformly distributed slots (equal offsets) around the waveguide broad-wall center line suffers from high side lobes $(\approx-12.6 \mathrm{~dB})$. After applying various tapering methods on the slots distribution, it was found out that utilizing the binomial distribution when calculating each slot offset result in a very low side lobe level $(<-22 \mathrm{~dB})$. The optimized slots offsets are shown in TABLE I.

TABLE I. - $40 \mathrm{~dB}$ Binomial tapering coefficients and corresponding slots offsets

\begin{tabular}{|c|c|c|}
\hline Slot No. & Binomial Coffs. & Displacement (mm) \\
\hline $1 \& 8$ & 1 & 0.5 \\
\hline $2 \& 7$ & 7 & 1.3 \\
\hline $3 \& 6$ & 21 & 2.3 \\
\hline $4 \& 5$ & 35 & 2.9 \\
\hline
\end{tabular}

\section{B. Augmented SWGAA}

It was noticed that the mutual coupling between slots is relatively high in the conventional SWGAA design. Placing inverted L-shaped parasitic dipoles elements beside the long sides of each slot, the dipoles will be electrically coupled due to the waveguide surface current. Each slot acts as magnetic dipole and the parasitic elements act as electric dipoles. This scenario is shown in Fig. 3. The simulated design is shown Fig. 4. Due to this augmentation, the SWGAA parameters have to be optimized to get the best performance at the desired frequency, e.g. the slot width has increased from $1.6 \mathrm{~mm}$ to $2.2 \mathrm{~mm}$. The measured and simulation results and performance enhancements are discussed in section III.

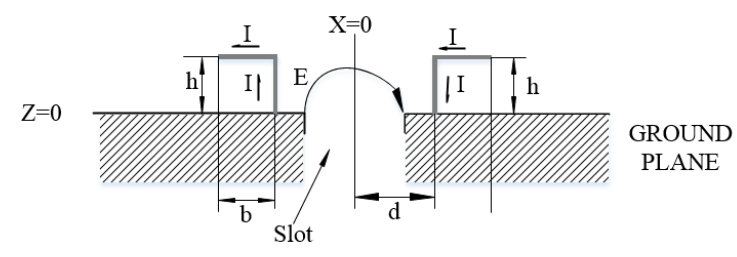

Fig. 3. Inverted L-shaped parasitic elements excitation phase

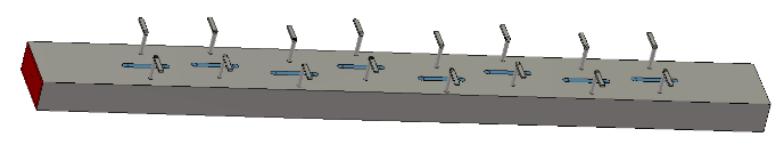

Fig. 4 SWGAA with parasitic elements

The dimensions of the parasitic elements are shown in TABLE II.

TABLE II. Parasitic elements dimensions

\begin{tabular}{|c|c|}
\hline Parameter & Value $(\mathrm{mm})$ \\
\hline$h$ & $\approx \lambda / 4$ \\
\hline$b$ & $\approx \lambda / 4$ \\
\hline$d$ & $\approx \lambda / 4$ \\
\hline Vertical part radius & 0.8 \\
\hline Horizontal part thickness & 0.8 \\
\hline
\end{tabular}

\section{RESULTS AND DISCUSSIONS}

It can be noticed from Fig. 5 that both antenna designs have a very good reflection coefficient (S11) at the chosen frequency.

Fig. 6 shows the azimuth radiation pattern for the two designs, both co-polarization and cross-polarization. The modified design of the SWGAA gain has improved by $2 \mathrm{~dB}$ while the antenna backlobe level is lowered by $2.1 \mathrm{~dB}$. As a result, the antenna front-to-back ratio has increased by approximately $4 \mathrm{~dB}$.

The azimuth beamwidth was $11.1^{\circ}$ before the augmentation and decreased to $11^{\circ}$ after placing the parasitic elements and optimize the design. On the other hand, as expected, the elevation plane radiation pattern shows a very wide beamwidth of about $87^{\circ}$, called fan beam. But, the proposed design has a $23^{\circ}$ narrower elevation plane beamwidth.

The sidelobe level of the proposed novel design is about 2.4 $\mathrm{dB}$ higher than that of the conventional SWGAA design. Even with this rise in the sidelobe level, the sidelobe level still $<-22$ $\mathrm{dB}$ which satisfy the antenna design requirements.

In Fig. 6, the design with parasitic elements has higher crosspolarization at $42^{\circ}$ in both sides of the main beam. The highest level in the cross-polarization is $-28.8 \mathrm{~dB}$ below the main lobe, which is below the required level of the $3 \mathrm{D}$ sidelobe level $(<-21$ $\mathrm{dB})$. On the other hand, the increase in the cross-polarization in the elevation plane is insignificant as can be seen in Fig. 7. The measured radiation pattern of the proposed design will presented in the conference. 


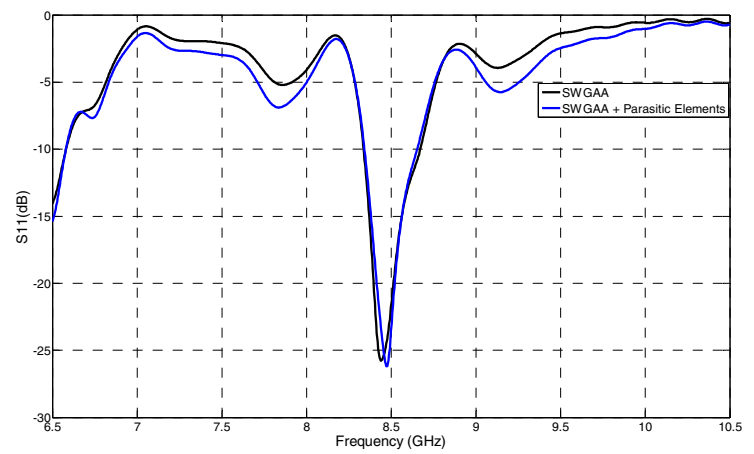

Fig. 5. Reflection coefficients for both designs

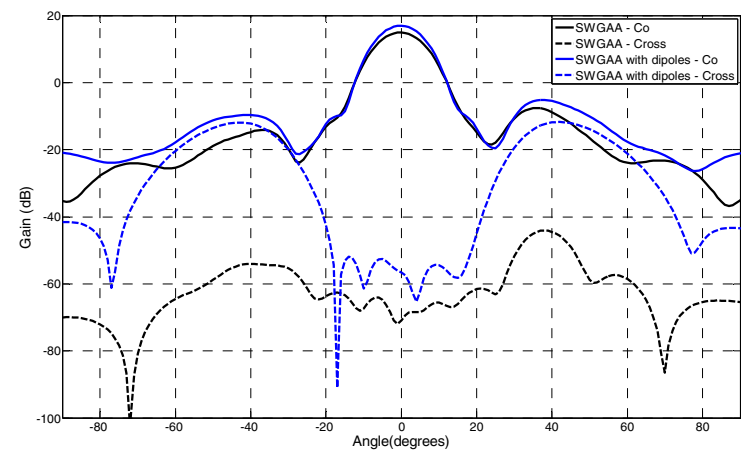

Fig. 6. Azimuth plane radiations patterns co- \& cross- polarization

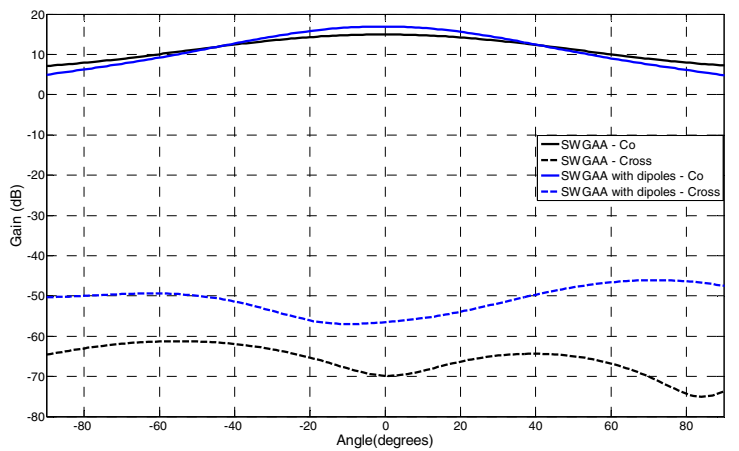

Fig. 7. Elevation plane radiation patterns co- \& cross- polarization

During the CST simulation, it was noticed that the mutual coupling between the slots in the conventional SWGAA is high, i.e. high surface current flowing between slots. The highest surface current, mutual coupling, occurred between the two middle slots. An H-plane probe was placed between the two middle slots for both designs, in the mid-point of the straight line connecting the two slots. It was observed that the mutual coupling between the two middle slots was reduced by approximately $30 \mathrm{~dB}$ in the proposed design, compared to the conventional SWGAA.

The two parasitic elements (dipoles) are placed along the line that passes through each slot center. Placing the dipoles in these locations allow them to get maximum excitation, as the voltage is highest at these locations, with opposite polarity in each side of a slot. As a result, the parasitic elements beside each slot act as an electric dipole.
The parasitic elements parameters play a critical role in achieving the enhanced SWGAA performance. So, these parameters require a carful optimization to achieve the best SWGAA performance enhancement.

\section{CONCLUSIONS}

A novel SWGAA design has been proposed and simulated. The performance enhancements that this design add to the conventional SWGAA are described and discussed. The increase in the $\mathrm{F} / \mathrm{B}$ ration and the high reduction in the slots mutual coupling form the most important SWGAA performance enhancement.

It is clear that the proposed design, due to the reduction in the elevation plane beamwidth, will lead to less number of stacked SWGAA to achieve narrower elevation beamwidth. The reduction in the mutual coupling between slots allow for higher transmission efficiency and increase the possibility of using such antenna in near field applications.

\section{REFERENCES}

[1] R. K. Gayen and S. Das, "Resonance and Parametric Analysis of Planar Broad-Wall Longitudinal Slot Array Antennas," International Journal of Research in Communication Engineering, Vols. 2, No. 3, pp. 13 - 20, 2012.

[2] W. H. Watson, "Resonant Slots," The Journal of the Institution of Electrical Engineers - Part IIIA: Radiation, vol. 93, no. 4, pp. 747-777, 1946.

[3] A. F. Stenenson, "Theory of Slots in Rectangular WaveGuides," Journal of Applied Physics, vol. 19, no. 1, pp. 24 - 38, 1947.

[4] H. Booker, "Slot aerials and their relation to complementary wire aerials (Babinet's principle)," Journal of the Institution of Electrical Engineers - Part IIIA: Radiolocation, vol. 93, no. 4, pp. 620 - 626, 1946.

[5] A. Clavin and W. W. Black, "DIPOLE AUGMENTED SLOT RADIATING ELEMENTS". United States Patent 3,594,806, 20 July 1971.

[6] A. Chlavin, "A New Antenna Feed Having Equal E- and H-Plane Patterns," Transactions of the IRE Professional Group on Antennas and Propagation, vol. 2, no. 3, pp. 113 - 119, 1954.

[7] A. Clavin, D. A Huebner and F. J. Kilburg, "An Improved Element for Use in Array Antennas," IEEE Trans. on Antenna and Propagation, Vols. AP-22, pp. 521 - 526, 1974.

[8] Computer Simulation Technology - MICROWAVE STUDIO user manual, Darmstadt, Germany: CST AG, 2015.

[9] R. S. Elliot, Antenna Theory and Design, New Jersy: John Wiley and Sons INC., 2003. 